\title{
The canonical contact structure on the space of oriented null geodesics of pseudospheres and products
}

\author{
Yamile Godoy and Marcos Salvai * \\ FaMAF - CIEM, Ciudad Universitaria, 5000 Córdoba, Argentina \\ ygodoy@famaf.unc.edu.ar,salvai@famaf.unc.edu.ar
}

\begin{abstract}
Let $N$ be a pseudo-Riemannian manifold such that $\mathcal{L}^{0}(N)$, the space of all its oriented null geodesics, is a manifold. B. Khesin and S. Tabachnikov introduce a canonical contact structure on $\mathcal{L}^{0}(N)$ (generalizing the definition given by R. Low in the Lorentz case), and study it for the pseudo-Euclidean space. We continue in that direction for other spaces.

Let $S^{k, m}$ be the pseudosphere of signature $(k, m)$. We show that $\mathcal{L}^{0}\left(S^{k, m}\right)$ is a manifold and describe geometrically its canonical contact distribution in terms of the space of oriented geodesics of certain totally geodesic degenerate hypersurfaces in $S^{k, m}$. Further, we find a contactomorphism with some standard contact manifold, namely, the unit tangent bundle of some pseudo-Riemannian manifold. Also, we express the null billiard operator on $\mathcal{L}^{0}\left(S^{k, m}\right)$ associated with some simple regions in $S^{k, m}$ in terms of the geodesic flows of spheres.

For $N$ the pseudo-Riemannian product of two complete Riemannian manifolds, we give geometrical conditions on the factors for $\mathcal{L}^{0}(N)$ to be a manifold and exhibit a contactomorphism with some standard contact manifold.
\end{abstract}

MSC 2010: 37D50, 53C22, 53C50, 53D10, 53D25, 58D10 53B30, 53C50.

Key words and phrases: contact manifold, null geodesic, space of geodesics, billiards Running title: The canonical contact structure on the space of null geodesics

\section{Introduction}

Let $N$ be a complete pseudo-Riemannian manifold. Let $\gamma_{u}$ denote the unique geodesic in $N$ with initial velocity $u$. Two null geodesics $\gamma_{u}$ and $\gamma_{v}$ are said to be equivalent if there exist $\lambda>0$ and $t \in \mathbb{R}$ such that $v=\lambda \dot{\gamma}_{u}(t)$. In particular, they have the same

*Partially supported by CONICET, FONCYT, SECYT (UNC). 
trajectory and orientation. We call $\mathcal{L}^{0}(N)$ the set of all equivalence classes of oriented null geodesics of $N$.

For $X \in T_{p} N$ we denote $\|X\|=\langle X, X\rangle$ and $|X|=\sqrt{|\langle X, X\rangle|}$. For $r=0,1$, let $T^{r} N=\{u \in T N \mid\|u\|=r, u \neq 0\}$.

By abuse of notation, we say that $\mathcal{L}^{0}(N)$ is a manifold if it admits a differentiable structure (not necessarily Hausdorff) such that the projection $\Pi: T^{0} N \rightarrow \mathcal{L}^{0}(N)$, $\Pi(u)=\left[\gamma_{u}\right]$, is a smooth submersion (throughout the paper, smooth means $\mathcal{C}^{\infty}$ ). This is not always the case, see for example the pseudo-Riemannian metric on the torus $T^{2}$ given in [8] such that the trajectory of each null geodesic is dense. Nevertheless, infinitesimal considerations at a fixed $[\gamma] \in \mathcal{L}^{0}(N)$ are always possible, for instance by means of Jacobi fields along $\gamma$.

B. Khesin and S. Tabachnikov introduce in [5] a canonical contact structure on $\mathcal{L}^{0}(N)$, provided that it is a manifold (generalizing the definition given in the Lorentz case by R. Low in [8]), and study it for the pseudo-Euclidean space. We continue in that direction for other spaces such as pseudospheres and some products.

Let $\mathbb{R}^{k+1, m}$ be the pseudo-Euclidean space of signature $(k+1, m)$, that is, $\mathbb{R}^{k+1} \times \mathbb{R}^{m}$ endowed with the inner product whose norm is given by $\|(u, v)\|=|u|^{2}-|v|^{2}$ (here, $|\cdot|$ denotes the norm of the canonical inner product on the Euclidean space). The pseudosphere of radius 1 in $\mathbb{R}^{k+1, m}$ is the hyperquadric

$$
S^{k, m}=\left\{p \in \mathbb{R}^{k+1, m} \mid\langle p, p\rangle=1\right\}=\left\{\left.(u, v) \in \mathbb{R}^{k+1, m}|| u\right|^{2}-|v|^{2}=1\right\},
$$

which is a hypersurface of $\mathbb{R}^{k+1, m}$ with induced metric of signature $(k, m)$. Notice that the Lorentz pseudosphere $S^{k, 1}$ is the de Sitter space. The null geodesics of $S^{k, m}$ are straight lines in $\mathbb{R}^{k+1, m}$ with initial velocity in $T^{0} S^{k, m}$. See other geometric properties of pseudospheres for example in [9].

In section 3 we show that $\mathcal{L}^{0}\left(S^{k, m}\right)$ is a manifold and it is contactomorphic to the unit tangent bundle of a certain pseudo-Riemannian manifold. Besides, we describe geometrically its canonical contact distribution in terms of the space of oriented geodesics of some totally geodesic degenerate hypersurfaces in $S^{k, m}$. In this section we also express the null billiard operator on $\mathcal{L}^{0}\left(S^{k, m}\right)$ associated with some simple regions in $S^{k, m}$ in terms of the geodesic flow of spheres.

Given $M$ and $N$ complete Riemannian manifolds, we consider on $M \times N$ the pseudoRiemannian metric whose norm is defined by $\|(u, v)\|=|u|_{M}^{2}-|v|_{N}^{2}$, for each $(u, v) \in$ $T_{(p, q)}(M \times N)$ and $(p, q) \in M \times N$. We denote this pseudo-Riemannian manifold by $M_{+} \times N_{-}$. In section 4 we prove that $\mathcal{L}^{0}\left(M_{+} \times N_{-}\right)$is a manifold if the geodesic flow of $M$ is free and proper. We also find conditions on $M$ for the existence of a contactomorphism between $\mathcal{L}^{0}\left(M_{+} \times N_{-}\right)$and $\mathcal{L}(M) \times T^{1} N$, where $\mathcal{L}(M)$ is the space of oriented geodesics of $M$.

Spaces of geodesics, their geometric structures and their applications have also been studied for instance in $[1,2,4,11,12,13]$. 


\section{Preliminaries}

As in the introduction, let $N$ be a complete pseudo-Riemannian manifold and $\mathcal{L}^{0}(N)$ the set of all equivalence classes of oriented null geodesic of $N$.

Let $\mathcal{A}=\mathrm{Aff}_{+}(\mathbb{R})$ be the Lie group of orientation preserving affine transformations of $\mathbb{R}$ and consider the right action from $\mathcal{A}$ on $T^{0} N$ given as follows: if $u \in T^{0} N$ and $g \in \mathcal{A}$,

$$
u \cdot g:=\left.\frac{d}{d t}\right|_{0} \gamma_{u}(g(t))
$$

If this action is free and proper, then $\mathcal{L}^{0}(N) \simeq T^{0} N / \mathcal{A}$ is a Hausdorff differentiable manifold such that the canonical projection $\Pi: T^{0} N \rightarrow \mathcal{L}^{0}(N)$ is a submersion (see for instance Proposition 2.3.8 of [10]).

Let $\pi: T N \rightarrow N$ be the canonical projection and for $r=0,1$ let $i: T^{r} N \hookrightarrow T N$ be the inclusion. Let $\theta$ and $\alpha$ be the canonical 1-forms on $T N$ and $T^{r} N$ respectively, that is, for $u \in T N$ and $\xi \in T_{u} T N$,

$$
\theta_{u}(\xi)=\left\langle u, d \pi_{u} \xi\right\rangle \quad \text { and } \quad \alpha=i^{*} \theta
$$

Definition. [5, 8] Let $N$ be a pseudo-Riemannian manifold such that $\mathcal{L}^{0}(N)$ is a manifold. The canonical contact distribution $\mathcal{D}$ on $\mathcal{L}^{0}(N)$ is well defined by

$$
\mathcal{D}_{\Pi(u)}=d \Pi_{u}\left(\operatorname{Ker} \alpha_{u}\right)
$$

for each $u \in T^{0} N$.

The canonical contact structure is presented here following the approach of [8], in a slightly different way as in the article [5] by Khesin and Tabachnikov (they define it in two steps via the space of scaled light-like geodesics, obtaining at the same time a symplectization of $\mathcal{L}^{0}(N)$ ).

\section{The canonical contact structure on $\mathcal{L}^{0}\left(S^{k, m}\right)$}

The following theorem is motivated by the fact that unit tangent bundles of pseudoRiemannian manifolds are among the standard examples of contact manifolds (with contact form as in (2)).

Let $S_{+}^{k} \times S_{-}^{m-1}$ be the manifold $S^{k} \times S^{m-1}$ with the pseudo-Riemannian metric such that for each $(x, y) \in T_{(u, v)}\left(S^{k} \times S^{m-1}\right),\|(x, y)\|=|x|^{2}-|y|^{2}$.

Theorem 1. The set $\mathcal{L}^{0}\left(S^{k, m}\right)$ is a manifold and if one considers on $\mathcal{L}^{0}\left(S^{k, m}\right)$ and $T^{1}\left(S_{+}^{k} \times S_{-}^{m-1}\right)$ the canonical contact structures, then the map

$$
F: T^{1}\left(S_{+}^{k} \times S_{-}^{m-1}\right) \rightarrow \mathcal{L}^{0}\left(S^{k, m}\right), \quad F((u, v),(x, y))=[\gamma]
$$

with $\gamma(t)=(x, y)+t(u, v)$, is a contactomorphism. 
Proof. First we prove that $\mathcal{L}^{0}\left(S^{k, m}\right)$ is a manifold. As explained above, since a straightforward computation yields that the action of $\mathcal{A}$ is clearly free, is suffices to check that the action is proper. In fact, let $\left(p_{n}, u_{n}\right)$ be a sequence converging to $(p, u)$ in $T^{0} S^{k, m}$ and let $\left(s_{n}, \lambda_{n}\right)$ be a sequence in $\mathbb{R} \rtimes \mathbb{R}_{+} \cong \mathcal{A}$ such that $\left(p_{n}, u_{n}\right) \cdot\left(s_{n}, \lambda_{n}\right)$ converges to $(q, v)$ in $T^{0} S^{k, m}$. We have to show that there exists a convergent subsequence of $\left(s_{n}, \lambda_{n}\right)$ in $\mathcal{A}$. The footpoints $p_{n}$ converge to $p$ in $S^{k, m}$ and as the null geodesics in $S^{k, m}$ are straight lines, for each $n \in \mathbb{N},\left(p_{n}, u_{n}\right) \cdot\left(s_{n}, \lambda_{n}\right)=\left(p_{n}+s_{n} u_{n}, \lambda_{n} u_{n}\right)$. Hence, by hypothesis, $\lambda_{n} u_{n} \rightarrow v$ and $p_{n}+s_{n} u_{n} \rightarrow q$ as well. Considering the canonical inner product $\langle$,$\rangle on \mathbb{R}^{k+1+m}$, since $u \neq 0$, we obtain that

$$
\lambda_{n} \rightarrow\langle v, u\rangle /|u|^{2} \quad \text { and } \quad s_{n} \rightarrow\langle q-p, u\rangle /|u|^{2} .
$$

Next, we verify that $F$ is a diffeomorphism. The map is well defined since given $(x, y) \in T_{(u, v)}^{1}\left(S_{+}^{k} \times S_{-}^{m-1}\right)$, we have that

$$
|u|^{2}=1=|v|^{2}, \quad\langle u, x\rangle=0=\langle v, y\rangle \text { and }|x|^{2}-|y|^{2}=1 .
$$

Then, $(x, y) \in S^{k, m},(u, v) \in(x, y)^{\perp}=T_{(x, y)} S^{k, m},\|(u, v)\|=0$ and $t \mapsto(x, y)+t(u, v)$ is a null geodesic in $S^{k, m}$. Thus, $F((u, v),(x, y)) \in \mathcal{L}^{0}\left(S^{k, m}\right)$.

Now, $F$ is smooth since all the spaces involved are (quotients of) embedded submanifolds of $E=\mathbb{R}^{k+1+m} \times \mathbb{R}^{k+1+m}$ and $g: E \rightarrow E, g((u, v),(x, y))=((x, y),(u, v))$, is obviously smooth and descends to $F$.

On the other hand, if $\gamma$ is a null geodesic in $S^{k, m}$, then $\gamma(t)=(x, y)+t(u, v)$ with $(x, y) \in S^{k, m}, 0 \neq(u, v) \perp(x, y)$ in $\mathbb{R}^{k+1, m}$ and $|u|^{2}-|v|^{2}=0$. So, we have that

$$
F^{-1}([\gamma])=\left(|u|^{-1}(u, v),(x, y)-|u|^{-2}\langle x, u\rangle(u, v)\right)
$$

and this is a smooth map.

Finally, we check that $F$ is a contactomorphism, that is $d F(\operatorname{Ker} \omega)=\mathcal{D}$, where $\mathcal{D}$ is defined in (3) and $\omega$ is the canonical contact form on $T^{1}\left(S_{+}^{k} \times S_{-}^{m-1}\right)$ as in (2).

Let $p: T^{1}\left(S_{+}^{k} \times S_{-}^{m-1}\right) \rightarrow S_{+}^{k} \times S_{-}^{m-1}$ be the canonical projection and let $f: T^{1}\left(S_{+}^{k} \times\right.$ $\left.S_{-}^{m-1}\right) \rightarrow T^{0} S^{k, m}$ be the restriction of $g$ defined above. Let $U=((u, v),(x, y)) \in$ $T^{1}\left(S_{+}^{k} \times S_{-}^{m-1}\right)$ and let $\xi \in \operatorname{Ker} \omega_{U}$. Since $F=\Pi \circ f$, we only have to verify that $d f_{U} \xi \in \operatorname{Ker} \alpha_{f(U)}$. For this, let $t \mapsto(c(t), z(t))$ be a curve in $T^{1}\left(S_{+}^{k} \times S_{-}^{m-1}\right)$ such that $c(0)=(u, v), z(0)=(x, y)$ and with initial velocity $\xi$.

By definition of $\omega$, we have that

$$
0=\omega_{U}(\xi)=\left\langle d p_{U} \xi, z(0)\right\rangle=\left\langle c^{\prime}(0), z(0)\right\rangle .
$$

Since $(z(t), c(t))=f(c(t), z(t)) \in T^{0} S^{k, m}$, it follows that $c(t) \perp z(t)$ in $\mathbb{R}^{k+1, m}$ for all $t$. Then,

$$
0=\left.\frac{d}{d t}\right|_{0}\langle c(t), z(t)\rangle=\left\langle c^{\prime}(0), z(0)\right\rangle+\left\langle c(0), z^{\prime}(0)\right\rangle .
$$

Therefore,

$$
\alpha_{f(U)}\left(d f_{U} \xi\right)=\left\langle d \pi_{f(U)}\left(d f_{U} \xi\right), c(0)\right\rangle=\left\langle d(\pi \circ f)_{U} \xi, c(0)\right\rangle=\left\langle z^{\prime}(0), c(0)\right\rangle=0 .
$$


Consequently, $d F_{U} \xi \in \mathcal{D}_{F(U)}$, and since both contact distributions have the same dimension, they are equal.

The following is an analogue of Proposition 2.6 (1) of [5].

Proposition 2. Let $\gamma(t)=p+t u$ be a null geodesic in $S^{k, m}$. Let $H$ be the totally geodesic degenerate hypersurface of $S^{k, m}$ containing the image of $\gamma$, given by $H=$ $u^{\perp} \cap S^{k, m}$ and let $\mathcal{L}(H)$ be the space of all oriented geodesics of $H$. If $\mathcal{D}$ is the canonical contact distribution on $\mathcal{L}^{0}\left(S^{k, m}\right)$, then, at the infinitesimal level,

$$
\mathcal{D}_{[\gamma]}=T_{[\gamma]} \mathcal{L}(H) .
$$

Proof. The statement is meant in the following sense (we do not address the question whether $\mathcal{L}(H)$ is a manifold): Given $X=d \Pi_{[\gamma]}(\xi) \in \mathcal{D}_{[\gamma]}$ (we recall that $\mathcal{D}$ is defined in (3)), there exists a variation by geodesics contained in $H$ whose associated Jacobi field along $\gamma$ satisfies $J(0)=d \pi_{u} \xi$ and $J^{\prime}(0)=K_{u}(\xi)$ (here $K_{u}: T_{u} T^{0} S^{k, m} \rightarrow T_{\pi(u)} S^{k, m}$ is the connection operator).

Specifically, since $\xi \in \operatorname{Ker} \alpha_{u} \subset T_{u} T^{0} S^{k, m}$ we have that $\left\langle d \pi_{u} \xi, u\right\rangle=0=\left\langle K_{u}(\xi), u\right\rangle$ and this implies that $d \pi_{u} \xi, K_{u}(\xi) \in T_{\pi(u)} H$. Let $c$ be a curve in $H$ such that $c(0)=\pi(u)$ and $c^{\prime}(0)=d \pi_{u} \xi$ and consider

$$
s \mapsto v(s)=\tau_{0}^{s}\left(u+s K_{u}(\xi)\right),
$$

where $\tau_{0}^{s}$ denotes the parallel transport along $c$ from 0 to $s$. Since $H$ is totally geodesic and $u+s K_{u}(\xi) \in T_{\pi(u)} H$ for all $s \in \mathbb{R}$, we obtain that $v(s) \in T_{c(s)} H$ and the image of $\gamma_{v(s)}$ is contained in $H$ for any $s$ (see for instance [9, page 125]). Besides, since

$$
v(0)=u \quad \text { and }\left.\quad \frac{D}{d s}\right|_{0} v(s)=K_{u}(\xi)
$$

then the Jacobi field $J(s)=\left.\frac{d}{d t}\right|_{0} \gamma_{v(s)}(t)$ along $\gamma$ has the desired properties.

\subsection{Billiards}

We recall the definition of the null billiard map (see Section 3 of [5]) in a special case. Let $N$ be a complete pseudo-Riemannian manifold and let $R$ be a region in $N$ with smooth nondegenerate boundary $M$. We require additionally that any null geodesic $\gamma$ intersecting the interior of $R$ satisfies that $\gamma(\mathbb{R}) \cap R=\gamma\left(\left[t_{0}, t_{1}\right]\right)$. We call $\mathfrak{L} \subset \mathcal{L}^{0}(N)$ the set of all oriented null geodesics intersecting the interior of $R$.

Let $\gamma$ be a null geodesic of $N$ such that $[\gamma] \in \mathfrak{L}$. Decompose $\dot{\gamma}\left(t_{1}\right)$ into its tangential and normal components, that is, $\dot{\gamma}\left(t_{1}\right)=u^{T}+u^{\perp}$ with $u^{T} \in T_{\gamma\left(t_{1}\right)} M$ and $u^{\perp} \in$ $\left(T_{\gamma\left(t_{1}\right)} M\right)^{\perp}$. The null billiard operator $B$ is well defined in the following way:

$$
B: \mathfrak{L} \rightarrow \mathfrak{L}, \quad B([\gamma])=\left[\gamma_{w}\right], \text { with } w=u^{T}-u^{\perp} .
$$

As in the pseudo-Euclidean case [5], the null billiard operator preserves the contact structure on $\mathcal{L}^{0}(N)$. For the sake of completeness, we include this fact as a proposition. 
Proposition 3. Let $N$ be a complete pseudo-Riemannian manifold and let $R$ be a region in $N$ as above. Then the canonical contact structure on $\mathcal{L}^{0}(N)$ is preserved by $B$.

Proof. Let $\ell \in \mathfrak{L}$ and $X \in \mathcal{D}_{\ell}$. By the definition of $\mathcal{L}^{0}(N)$ we can take $u \in T^{0} N$ such that $\Pi(u)=\ell$ and $\pi(u) \in M$. There exists $\eta \in \operatorname{Ker} \alpha_{u}$ such that $d \Pi_{u} \eta=X$. Since $T_{\pi(u)} N=\mathbb{R} u+T_{\pi(u)} M$, then $d \pi_{u} \eta=\lambda u+v$, with $v \in T_{\pi(u)} M$ and $\lambda \in \mathbb{R}$. Let $\tau: T_{u} T N \rightarrow T_{\pi(u)} N \times T_{\pi(u)} N$ be the isomorphism given by $\tau(\xi)=\left(d \pi_{u} \xi, K_{u}(\xi)\right)$. Thus, $\xi=\tau^{-1}\left(v, K_{u}(\eta)\right)$ satisfies that $\xi \in \operatorname{Ker} \alpha_{u}, d \Pi_{u} \xi=X$ and $d \pi_{u} \xi \in T_{\pi(u)} M$. Let $c$ be a curve in $M$ with initial velocity $d \pi_{u} \xi$. Since $\left.\pi\right|_{T^{0} N}$ is a submersion, there exists a curve $t \mapsto u(t)$ in $T^{0} N$ such that $u(0)=u, u^{\prime}(0)=\xi$ and $\pi(u(t))=c(t)$. So,

$$
0=\alpha_{u}(\xi)=\left\langle u(0), d \pi_{u(0)} u^{\prime}(0)\right\rangle=\left\langle u(0), c^{\prime}(0)\right\rangle .
$$

We decompose $u(t)=u^{T}(t)+u^{\perp}(t)$, where $u^{T}(t) \in T_{c(t)} M$ and $u^{\perp}(t) \in\left(T_{c(t)} M\right)^{\perp}$ (we recall that $M$ is supposed to be nondegenerate). Taking $\ell(t)=\Pi(u(t))$, we have

$$
d B_{\ell} X=\left.\frac{d}{d t}\right|_{0} B(\ell(t))=\left.\frac{d}{d t}\right|_{0} \Pi\left(u^{T}(t)-u^{\perp}(t)\right) .
$$

We observe that $\pi\left(u^{T}(t)-u^{\perp}(t)\right)=c(t)$. Thus, to see that $d B_{\ell} X \in \mathcal{D}_{B(\ell)}$ we only have to show that

$$
\left\langle u^{T}(0)-u^{\perp}(0), c^{\prime}(0)\right\rangle=0 .
$$

But, by (6) and the fact that $c^{\prime}(0) \in T_{c(0)} M$, we obtain that $\left\langle u^{T}(0), c^{\prime}(0)\right\rangle=0$, and this implies that (7) holds.

Finally, since $\mathcal{D}$ has constant dimension and $d B_{\ell}$ is nonsingular, it follows that $d B_{\ell} \mathcal{D}_{\ell}=\mathcal{D}_{B(\ell)}$.

For $c>0$, let $R_{c}$ be the region in $S^{k, m}$ given by

$$
R_{c}=\left\{(u, v) \in S^{k, m}|| v \mid \leq c\right\},
$$

with boundary $M_{c}=\left\{(u, v) \in S^{k, m}|| v \mid=c\right\}$, which is nondegenerate since $V(u, v)=$ $\left(c^{2} u,\left(1+c^{2}\right) v\right)$ is an outside pointing normal light-like vector field.

We write the null billiard operator $B$ via $F$ of Theorem 1, in terms of the geodesic flow of spheres. For this, we consider the map

$$
i: T^{1}\left(S_{+}^{k} \times S_{-}^{m-1}\right) \rightarrow T S^{k} \times T S^{m-1}, \quad i((u, v),(x, y))=((u, x),(v, y)) .
$$

As before, we call $\mathfrak{L}$ the set of all oriented null geodesics in $S^{k, m}$ that intersect the interior of $R_{c}$ and denote $L=i \circ F^{-1}(\mathfrak{L}) \subset T S^{k} \times T S^{m-1}$.

We call $\varphi$ and $\psi$ the geodesic flows of $S^{k}$ and $S^{m-1}$, respectively.

Proposition 4. Let $\tilde{B}: L \rightarrow L$ be the conjugate of the null billiard operator on $\mathfrak{L}$ by the map $i \circ F^{-1}$. Then,

$$
\tilde{B}((u, x),(v, y))=\left(|x| \varphi_{2 \theta_{x}}(u, x /|x|),|y| \psi_{2 \theta_{y}}(v, y /|y|)\right),
$$

where $\theta_{x}, \theta_{y} \in\left(-\frac{\pi}{2}, 0\right]$ are such that $|x| \tan \theta_{x}=-\sqrt{c^{2}-|y|^{2}}=|y| \tan \theta_{y}$. 
Proof. Let $((u, x),(v, y)) \in L$. Using (4), we find that $t_{1}=\sqrt{c^{2}-|y|^{2}}$ is as in the definition of the null billiard operator. So, we have that $F((u, v),(x, y))=[\gamma]$ with $\gamma(t)=(x, y)+t_{1}(u, v)+t(u, v)$ and we can decompose the vector $(u, v)$ into its tangential and normal parts at $\gamma(0)$. Indeed,

$$
\begin{aligned}
& \qquad(u, v)^{T}=\left(\frac{1}{1+c^{2}}\left(|x|^{2} u-t_{1} x\right), \frac{1}{c^{2}}\left(|y|^{2} v-t_{1} y\right)\right) \\
& \text { and } \quad(u, v)^{\perp}=\left(\frac{t_{1}}{1+c^{2}}\left(t_{1} u+x\right), \frac{t_{1}}{c^{2}}\left(t_{1} v+y\right)\right) .
\end{aligned}
$$

Then, by definition of $B$ and using the expression for the inverse of $F$ given in (5), we obtain that $\tilde{B}((u, x),(v, y))=\left(\left(u^{\prime}, x^{\prime}\right),\left(v^{\prime}, y^{\prime}\right)\right)$, where

$$
\begin{aligned}
\left(u^{\prime}, x^{\prime}\right) & =\left(\frac{|x|^{2}-t_{1}^{2}}{1+c^{2}} u-\frac{2 t_{1}|x|}{1+c^{2}} \frac{x}{|x|},|x|\left(\frac{2 t_{1}|x|}{1+c^{2}} u+\frac{|x|^{2}-t_{1}^{2}}{1+c^{2}} \frac{x}{|x|}\right)\right) \\
& =|x| \varphi_{2 \theta_{x}}(u, x /|x|),
\end{aligned}
$$

with $\theta_{x}$ such that $\tan \theta_{x}=-t_{1} /|x|$, and

$$
\begin{aligned}
\left(v^{\prime}, y^{\prime}\right) & =\left(\frac{|y|^{2}-t_{1}^{2}}{c^{2}} v-\frac{2 t_{1}|y|}{c^{2}} \frac{y}{|y|},|y|\left(\frac{2 t_{1}|y|}{c^{2}} v+\frac{|y|^{2}-t_{1}^{2}}{c^{2}} \frac{y}{|y|}\right)\right) \\
& =|y| \psi_{2 \theta_{y}}(v, y /|y|),
\end{aligned}
$$

with $\theta_{y}$ such that $\tan \theta_{y}=-t_{1} /|y|$.

Corollary 5. (Lorentz case) Let $\tilde{B}$ be the conjugate of the null billiard operator on $\mathcal{L}^{0}\left(S^{k, 1}\right)$ by the identifications $\mathcal{L}^{0}\left(S^{k, 1}\right) \simeq T^{1}\left(S_{+}^{k} \times S_{-}^{0}\right) \simeq T^{1} S^{k} \times\{-1,1\}$, then

$$
\tilde{B}((u, x), \varepsilon)=(\varphi-2 \arctan (c)(u, x),-\varepsilon),
$$

where $u \in S^{k}, x \perp u$ and $\varepsilon= \pm 1$.

\section{The canonical contact structure on $\mathcal{L}^{0}\left(M_{+} \times N_{-}\right)$}

Let $M$ and $N$ be complete Riemannian manifolds. Let $M_{+} \times N_{-}$be the manifold $M \times N$ with the pseudo-Riemannian metric whose norm is defined by $\|(u, v)\|=|u|_{M}^{2}-|v|_{N}^{2}$, for each $(u, v) \in T_{(p, q)}(M \times N)$ and $(p, q) \in M \times N$.

Let $\mathcal{L}(M)$ be the space of oriented geodesics of $M$, that is, the quotient of $T^{1} M$ by the action of $\mathbb{R}$ on it determined by the geodesic flow of $M$.

We call $p_{1}, p_{2}$ the projections of $\mathcal{L}(M) \times T^{1} N$ onto the first and second factors, respectively, and let $\alpha_{1}$ and $\alpha_{2}$ be the canonical 1-forms on $T^{1} M$ and $T^{1} N$, respectively, defined as in (2). 
Theorem 6. Let $M$ and $N$ be complete Riemannian manifolds such that the geodesic flow of $M$ is free and proper. Then, $\mathcal{L}^{0}\left(M_{+} \times N_{-}\right)$is a manifold. Suppose additionally that there exists a smooth global section $S: \mathcal{L}(M) \rightarrow T^{1} M$. Then $\theta_{S}=p_{1}^{*} S^{*} \alpha_{1}-p_{2}^{*} \alpha_{2}$ is a contact 1 -form on $\mathcal{L}(M) \times T^{1} N$ and the map

$$
G: \mathcal{L}(M) \times T^{1} N \rightarrow \mathcal{L}^{0}\left(M_{+} \times N_{-}\right), \quad G([\sigma], v)=\left[\left(\gamma_{S([\sigma])}, \gamma_{v}\right)\right]
$$

is a contactomorphism, where $\mathcal{L}^{0}\left(M_{+} \times N_{-}\right)$is endowed with its canonical contact structure.

Proof. First, notice that $\mathcal{L}(M)=T^{1} M / \mathbb{R}$ is a manifold since the geodesic flow of $M$ is free and proper. Now, $\mathcal{L}^{0}\left(M_{+} \times N_{-}\right)$is also a manifold since the right action from $\mathcal{A}$ on $T^{0}\left(M_{+} \times N_{-}\right)$defined in (1) turns out to be proper and free. Indeed, the action is free due to the fact that the geodesics have constant speed and the geodesic flow of $M$ is free. On the other hand, given a sequence $\left(u_{n}, v_{n}\right)$ converging to $(u, v)$ in $T^{0}\left(M_{+} \times N_{-}\right)$and a sequence $\left(s_{n}, \lambda_{n}\right)$ in $\mathbb{R} \rtimes \mathbb{R}_{+} \cong \mathcal{A}$ such that the sequence $\left(u_{n}, v_{n}\right) \cdot\left(s_{n}, \lambda_{n}\right)=\left(\lambda_{n} \dot{\gamma}_{u_{n}}\left(s_{n}\right), \lambda_{n} \dot{\gamma}_{v_{n}}\left(s_{n}\right)\right)$ converges to $(z, w)$ in $T^{0}\left(M_{+} \times N_{-}\right)$, then we have that

$$
\lambda_{n} \dot{\gamma}_{u_{n}}\left(s_{n}\right) \rightarrow z \quad \text { and } \quad u_{n} \rightarrow u
$$

in $T M$. So,

$$
\lambda_{n}\left|\dot{\gamma}_{u_{n}}\left(s_{n}\right)\right| \rightarrow|z| \text { and } \quad\left|\dot{\gamma}_{u_{n}}\left(s_{n}\right)\right| \rightarrow|u| \neq 0,
$$

and then $\lambda_{n} \rightarrow|z| /|u|$. Furthermore, since

$$
\dot{\gamma}_{u_{n} /\left|u_{n}\right|}\left(\left|u_{n}\right| s_{n}\right)=\left|u_{n}\right|^{-1} \dot{\gamma}_{u_{n}}\left(s_{n}\right) \quad \text { and } \quad \dot{\gamma}_{u_{n}}\left(s_{n}\right)=\lambda_{n}^{-1}\left(\lambda_{n} \dot{\gamma}_{u_{n}}\left(s_{n}\right)\right) \rightarrow|u| z /|z| \text {, }
$$

we obtain that

$$
\dot{\gamma}_{u_{n} /\left|u_{n}\right|}\left(\left|u_{n}\right| s_{n}\right) \rightarrow z /|z|
$$

in $T^{1} M$. Since the sequence $u_{n} /\left|u_{n}\right|$ converges to $u /|u|$ in $T^{1} M$ and the geodesic flow of $M$ is proper, there exits a subsequence $\left|u_{n_{j}}\right| s_{n_{j}}$ converging to some $s$ in $\mathbb{R}$. Therefore, $\left(s_{n_{j}}, \lambda_{n_{j}}\right) \rightarrow(s /|u|,|z| /|u|)$ in $\mathcal{A}$, and so the action is proper.

To verify that $\left(\mathcal{L}(M) \times T^{1} N, \theta_{S}\right)$ is a contact manifold we show that $G$ is a diffeomorphism such that $d G\left(\operatorname{Ker} \theta_{S}\right)=\mathcal{D}$, where $\mathcal{D}$ is the contact distribution as in (3).

Let $h: T^{1} M \times T^{1} N \rightarrow T^{0}\left(M_{+} \times N_{-}\right)$be the canonical inclusion. Since $G=$ $\Pi \circ h \circ(S \times$ id $)$ and any of these maps is smooth, we obtain that $G$ is smooth.

Let $\pi_{M}: T^{1} M \rightarrow \mathcal{L}(M)$ be the canonical projection. Under the hypothesis on the geodesic flow of $M,\left(T^{1} M, \pi_{M}, \mathcal{L}(M)\right)$ is an $\mathbb{R}$-principal bundle (see for instance [10, Proposition 2.3.8 (iii)]). So, there exists a smooth map $x: T^{1} M \rightarrow \mathbb{R}$ such that $S\left(\pi_{M}(u)\right)=\dot{\gamma}_{u}(x(u))$. Then, if $\gamma$ and $\sigma$ are geodesics in $M$ and $N$, respectively, such that $[(\gamma, \sigma)] \in \mathcal{L}^{0}\left(M_{+} \times N_{-}\right)$, we have that

$$
G^{-1}: \mathcal{L}^{0}\left(M_{+} \times N_{-}\right) \rightarrow \mathcal{L}(M) \times T^{1} N, \quad G^{-1}([(\gamma, \sigma)])=\left(\left[\gamma_{u}\right], \dot{\gamma}_{v}(x(u))\right),
$$


where $u=\dot{\gamma}(0) /|\dot{\gamma}(0)| \in T^{1} M$ and $v=\dot{\sigma}(0) /|\dot{\sigma}(0)| \in T^{1} N$. Since $G^{-1} \circ \pi_{M}$ is smooth and $\pi_{M}$ is a submersion, it follows that $G^{-1}$ is a smooth map. Therefore, $G$ is a diffeomorphism.

Finally, we check that $d G\left(\operatorname{Ker} \theta_{S}\right)=\mathcal{D}$. For this, let $p=([\sigma], v) \in \mathcal{L}(M) \times T^{1} N$ and take $(\xi, \eta) \in \operatorname{Ker}\left(\theta_{S}\right)_{p}$. Let $t \mapsto\left(\ell_{t}, v_{t}\right)$ be a curve in $\mathcal{L}(M) \times T^{1} N$ such that $\left(\ell_{0}, v_{0}\right)=p$ and $\left(\ell_{0}^{\prime}, v_{0}^{\prime}\right)=(\xi, \eta)$. Since $G\left(\ell_{t}, v_{t}\right)=\Pi\left(S\left(\ell_{t}\right), v_{t}\right)$, then

$$
d G_{p}(\xi, \eta)=\left.\frac{d}{d t}\right|_{0} G\left(\ell_{t}, v_{t}\right)=\left.d \Pi_{(S([\sigma]), v)} \frac{d}{d t}\right|_{0}\left(S\left(\ell_{t}\right), v_{t}\right) .
$$

By definition of $\mathcal{D}$, we only have to verify that $X=\left.\frac{d}{d t}\right|_{0}\left(S\left(\ell_{t}\right), v_{t}\right)$ is in $\operatorname{Ker} \alpha_{(S([\sigma]), v)}$. If we call $\pi^{1}: T^{1} M \rightarrow M$ and $\pi^{2}: T^{1} N \rightarrow N$ the canonical projections, we have that

$$
d \pi_{(S([\sigma]), v)} X=\left(d \pi_{S([\sigma])}^{1}\left(d S_{[\sigma]} \xi\right), d \pi_{v}^{2}(\eta)\right) .
$$

Then,

$$
\begin{aligned}
\alpha_{(S([\sigma]), v)}(X) & =\left\langle(S([\sigma]), v), d \pi_{(S([\sigma]), v)} X\right\rangle \\
& =\left\langle S([\sigma]), d \pi_{S([\sigma])}^{1}\left(d S_{[\sigma]} \xi\right)\right\rangle_{M}-\left\langle v, d \pi_{v}^{2}(\eta)\right\rangle_{N} \\
& =\left(S^{*} \alpha_{1}\right)_{[\sigma]}(\xi)-\left(\alpha_{2}\right)_{v}(\eta) \\
& =\left(p_{1}^{*} S^{*} \alpha_{1}-p_{2}^{*} \alpha_{2}\right)_{(S([\sigma]), v)}(\xi, \eta) \\
& =\left(\theta_{S}\right)_{p}(\xi, \eta)=0 .
\end{aligned}
$$

Hence, $d G_{p}(\xi, \eta) \in \mathcal{D}_{G(p)}$. Since $d G\left(\operatorname{Ker} \theta_{S}\right)$ and $\mathcal{D}$ have the same dimension, we obtain their equality. Consequently, since $\mathcal{D}$ is a contact distribution, $\theta_{S}$ is a contact 1 -form on $\mathcal{L}(M) \times T^{1} N$ and $G$ is a contactomorphism.

Example 1. Writing $\mathbb{R}^{n, k}=\mathbb{R}_{+}^{n} \times \mathbb{R}_{-}^{k}$ one has $\mathcal{L}^{0}\left(\mathbb{R}^{n, k}\right) \simeq \mathcal{L}\left(\mathbb{R}^{n}\right) \times T^{1} \mathbb{R}^{k} \simeq T S^{n-1} \times$ $\mathbb{R}^{k} \times S^{k-1}$. Proposition $2.6(2)$ in [5] gives another presentation of $\mathcal{L}^{0}\left(\mathbb{R}^{n, k}\right)$, in terms of 1-jets, which has the advantage of being natural.

Example 2. If $M$ is either a Hadamard manifold or the paraboloid of revolution $\left\{\left(x, y, x^{2}+y^{2}\right) \mid x, y \in \mathbb{R}\right\}$, then $\mathcal{L}(M)$ is a manifold and has a smooth section into $T^{1} M$, and hence it satisfies the hypotheses of Theorem 6 .

Suppose first that $M$ is a Hadamard manifold. The geodesic flow of $M$ is free since the exponential map is a diffeomorphism at every point. Besides, given a sequence $\left(p_{n}, v_{n}\right)$ converging to $(p, v)$ in $T^{1} M$ and a sequence $t_{n}$ in $\mathbb{R}$ such that $\left(\gamma_{v_{n}}\left(t_{n}\right), \dot{\gamma}_{v_{n}}\left(t_{n}\right)\right)$ converges to $(q, u)$, we have that $d\left(p_{n}, \gamma_{v_{n}}\left(t_{n}\right)\right)=\left|t_{n}\right|$, because geodesics in $M$ minimize the distance. Since the distance is a continuous map, it follows that $\left|t_{n}\right| \rightarrow d(p, q)$. Then the sequence $t_{n}$ has a convergent subsequence and the geodesic flow of $M$ is proper. Therefore, $\mathcal{L}(M)$ is a manifold.

Fixing $p \in M$, let $H: T_{p}^{1} M \rightarrow \mathcal{L}(M)$ be the map defined as follows: Let $X \in$ $T_{p}^{1} M$ and $Y \in T_{p} M$ with $X \perp Y$, then $H(X, Y)$ is the oriented geodesic with initial point $\exp _{p}(Y)$ and initial velocity the parallel transport of $X$ along the geodesic $t \mapsto$ 
$\exp _{p}(t X)$. Proposition 4.14 of [3] asserts that $H$ is a diffeomorphism. Thus, there exists a global section from $\mathcal{L}(M)$ into $T^{1} M$, namely, $S$ assigns to each oriented unit speed geodesic of $M$ its velocity at the closest point to $p$.

Now, let $M$ be the paraboloid of revolution. The geodesic flow $\varphi_{t}$ is free since $M$ has no periodic geodesics (see [6, Example 2.9.2]). Next, we show that it is proper. Suppose that $u_{n} \rightarrow u$ and $\varphi_{t_{n}}\left(u_{n}\right) \rightarrow z$ in $T^{1} M$. Let $c>0$ such that the footpoints of $u$ and $z$ belong to the interior of $C=\{p \in M \mid z \leq c\}$. Hence, for $n \geq N$ the footpoints of $u_{n}$ and $\varphi_{t_{n}}\left(u_{n}\right)$ also belong to the interior of $C$. Now, again by [6, Example 2.9.2], $C$ is totally convex. Hence, by Proposition 2.9.14 in [6], there exists $L>0$ such that every geodesic segment in $C$ has length $\leq L$. In particular, $\left|t_{n}\right| \leq L$, since $\left|t_{n}\right|$ is the length of the geodesic segment $\left.\gamma_{u_{n}}\right|_{I_{n}}$, where $I_{n}=\left[0, t_{n}\right]$ for $t_{n}>0$ and $I_{n}=\left[t_{n}, 0\right]$ for $t_{n}<0$. Therefore, $t_{n}$ has a convergent subsequence.

The existence of a smooth global section is proved in an analogous way as for a Hadamard manifold. Notice that each geodesic in the paraboloid which is not a meridian has an infinite number of self-intersections.

\section{References}

[1] D. Alekseevsky, B. Guilfoyle and W. Klingenberg, On the geometry of spaces of oriented geodesics. Ann. Global Anal. Geom. 40 (2011), 389-409.

[2] J. Beem and P. Parker, The space of geodesics. Geom. Dedicata 38 (1991), 87-99.

[3] J. Beem, R. Low and P. Parker, Spaces of geodesics: products, coverings, connectedness. Geom. Dedicata 59 (1996), 51-64.

[4] B. Guilfoyle and W. Klingenberg, An indefinite Kähler metric on the space of oriented lines. J. London Math. Soc. 72 (2005), 497-509.

[5] B. Khesin and S. Tabachnikov, Pseudo-Riemannian geodesics and billiards. Adv. Math. 221 (2009), 1364-1396.

[6] W. Klingenberg, Riemannian Geometry. Walter de Gruyter 1982.

[7] R. Low, The geometry of the space of null geodesics. J. Math. Phys. 30 (1989), 809-811.

[8] R. Low, The space of null geodesics. Nonlinear Anal. 47 (2001), 3005-3017.

[9] B. O’Neill, Semi-Riemannian geometry with applications to relativity. Academic Press 1983.

[10] J. Ortega and T. Ratiu, Momentum maps and Hamiltonian reduction. Progress in Mathematics 222, Springer 2004. 
[11] M. Salvai, On the geometry of the space of oriented lines of Euclidean space. Manuscr. Math. 118 (2005), 181-189.

[12] M. Salvai, On the geometry of the space of oriented lines of the hyperbolic space. Glasgow Math. J. 49 (2007), 357-366.

[13] M. Salvai, Global smooth fibrations of $\mathbb{R}^{3}$ by oriented lines. Bull. London Math. Soc. 41 (2009), 155-163. 